\title{
Clinical relevance of short-term follow-up of unruptured intracranial aneurysms
}

\author{
Rob Molenberg, BSc, ${ }^{1}$ Marlien W. Aalbers, MD, PhD, ${ }^{1}$ Jan D. M. Metzemaekers, MD, PhD, ${ }^{1}$ \\ Aryan Mazuri, MD, ${ }^{3}$ Gert-Jan Luijckx, MD, PhD, ${ }^{2}$ Rob J. M. Groen, MD, PhD, ${ }^{1}$ \\ Maarten Uyttenboogaart, MD, PhD, ${ }^{2,3}$ and J. Marc C. van Dijk, MD, PhD ${ }^{1}$
}

Departments of ${ }^{1}$ Neurosurgery, ${ }^{2}$ Neurology, and ${ }^{3}$ Radiology, University of Groningen, University Medical Center Groningen, Groningen, The Netherlands

\begin{abstract}
OBJECTIVE Unruptured intracranial aneurysms are common incidental findings on brain imaging. Short-term follow-up for conservatively treated aneurysms is routinely performed in most cerebrovascular centers, although its clinical relevance remains unclear. In this study, the authors assessed the extent of growth as well as the rupture risk during shortterm follow-up of conservatively treated unruptured intracranial aneurysms. In addition, the influence of patient-specific and aneurysm-specific factors on growth and rupture risk was investigated.

METHODS The authors queried their prospective institutional neurovascular registry to identify patients with unruptured intracranial aneurysms and short-term follow-up imaging, defined as follow-up MRA and/or CTA within 3 months to 2 years after initial diagnosis. Medical records and questionnaires were used to acquire baseline information. The authors measured aneurysm size at baseline and at follow-up to detect growth. Rupture was defined as a CT scan-proven and/ or CSF-proven subarachnoid hemorrhage (SAH).
\end{abstract}

RESULTS A total of 206 consecutive patients with 267 conservatively managed unruptured aneurysms underwent short-term follow-up at the authors' center. Seven aneurysms (2.6\%) enlarged during a median follow-up duration of 1 year (range $0.3-2.0$ years). One aneurysm $(0.4 \%)$ ruptured 10 months after initial discovery. Statistically significant risk factors for growth or rupture were autosomal-dominant polycystic kidney disease (RR 8.3, 95\% $\mathrm{Cl} 2.0-34.7$ ), aspect ratio $>1.6$ or size ratio $>3$ ( $\mathrm{RR} 10.8,95 \% \mathrm{Cl} 2.2-52.2)$, and initial size $\geq 7 \mathrm{~mm}(\mathrm{RR} 10.7,95 \% \mathrm{Cl} 2.7-42.8)$.

CONCLUSIONS Significant growth of unruptured intracranial aneurysms may occur in a small proportion of patients during short-term follow-up. As aneurysm growth is associated with an increased risk of rupture, the authors advocate that short-term follow-up is clinically relevant and has an important role in reducing the risk of a potential SAH.

https://thejns.org/doi/abs/10.3171/2019.4.FOCUS1995

KEYWORDS unruptured intracranial aneurysm; growth; follow-up; risk factors; subarachnoid hemorrhage

$\mathrm{U}$ NRUPTURED intracranial aneurysms are present in approximately $3 \%$ of the general population and represent the most common incidentally found arterial anomaly on brain imaging. ${ }^{3,24}$ Current clinical management of unruptured aneurysms focuses on identifying and treating aneurysms with a high estimated risk of rupture, in order to prevent the occurrence of a subarachnoid hemorrhage (SAH). Prevention of an SAH is crucial, since it is associated with significant morbidity and mortality and frequently affects relatively young individuals. ${ }^{17}$ On the other hand, the treatment-associated risks frequently outweigh the estimated risk of rupture, especially after introduction of the PHASES score? This is particularly true for patients younger than 70 years with aneurysms of the internal carotid artery or middle cerebral artery smaller than $7 \mathrm{~mm}$, which-even when assuming a high-risk profile with hypertension and a previous $\mathrm{SAH}-$ harbor a small estimated rupture risk of approximately $1 \%$ per year. ${ }^{7}$ As a result, a considerable group of patients is managed conservatively with serial follow-up imaging.

Growth of an aneurysm during follow-up increases the risk of rupture and is therefore considered as an indication for elective treatment. ${ }^{22}$ Literature regarding aneurysm growth and rupture during follow-up shows that many neurovascular centers initially recommend short-term follow-up imaging at 6 months to 1 year after diagnosis. ${ }^{6,8,10-12,14-16,18,20,22,25}$ Remarkably, although short-term follow-up is routinely advised, the extent of aneurysm growth 
on such a short-term, and thus the clinical relevance of short-term follow-up, is unclear.

The primary objectives of this study were to assess the extent of aneurysm growth and rupture risk during shortterm follow-up in a group of patients with conservatively treated unruptured intracranial aneurysms. In addition, the association between patient- and aneurysm-specific factors and both aneurysm growth and rupture was evaluated.

\section{Methods \\ Patient Selection}

The study protocol was approved by the local institutional research ethics board, and informed consent was obtained as required. Our prospectively maintained neurovascular registry was queried to identify patients with unruptured saccular intracranial aneurysms between 1998 and 2017. Patients at least 18 years old with one or more unruptured intracranial aneurysms were included. We defined short-term follow-up as follow-up imaging between 3 months and 2 years after initial evaluation. Extradural aneurysms (e.g., located in the cavernous sinus) and aneurysms that were treated during follow-up were excluded, as well as patients for whom follow-up imaging was not available.

\section{Patient Data}

Patients' medical records and a questionnaire were used to collect the following baseline characteristics: age and sex of the patient at the time of initial evaluation, as well as a history of SAH from a different aneurysm, aneurysm multiplicity, cigarette smoking, use of acetylsalicylic acid, familial intracranial aneurysms, hypertension, autosomal-dominant polycystic kidney disease (ADPKD), and alcohol abuse. If a patient did not return the questionnaire, the patient's medical record was reviewed for baseline information. Hypertension was considered present if a patient had a previous diagnosis of hypertension or used antihypertensive medication. A positive familial predisposition was considered present if at least 2 first-degree relatives experienced intracranial aneurysms or SAHs. Alcohol abuse was defined as the weekly consumption of at least $300 \mathrm{~g}$ ethanol. We contacted the general practitioner of patients who did not return the questionnaire to verify whether the patient was still alive and to exclude possible rupture of the aneurysm. Study data were collected and managed using REDCap (Research Electronic Data Capture) electronic data capture tools hosted at our institution. ${ }^{9}$

\section{Aneurysm Measurements}

We measured the size of all aneurysms at baseline and at follow-up. Imaging studies consisted mostly of MRA with 3D time-of-flight sequences with 0.6-mm slice thickness, and contrast-enhanced CTA with $0.75-\mathrm{mm}$ slice thickness. If patients underwent multiple follow-up scanning sessions within the interval for short-term follow-up, the most recent scan was used as the follow-up scan. We used syngo.via radiological software (version VB20A, Siemens Healthcare) to perform all measurements. An- eurysm height, width, and neck diameter were measured. We defined height as the largest distance from the center of the neck to the tip of the aneurysm dome. Aneurysm width was measured perpendicular to the height. Aspect ratio was calculated by dividing the maximum height, measured perpendicular to the neck of the aneurysm, by the neck diameter (Fig. 1). ${ }^{21}$ Size ratio was calculated by dividing the maximum height of the aneurysm by the average parent artery diameter. ${ }^{4}$ All aneurysm measurements were performed by one author (R.M.). The findings of the researcher were always compared to the findings of the neuroradiologist who reviewed the imaging during follow-up. The measurements of enlarged aneurysms were also assessed by a second reviewer (M.W.A.). When there was a discrepancy between the assessment of the reviewer and the neuroradiologist, an experienced neuro-interventionalist (M.U.) reviewed the measurements and made a final decision.

\section{Outcome}

The primary outcomes were growth or rupture during follow-up. Aneurysm growth was defined as either an increase in size of $\geq 1 \mathrm{~mm}$ in at least one direction or a change from a regular to an irregular shape. ${ }^{1}$ The latter was defined as the presence of blebs, multiple lobes, or wall protrusions, as previously described by Backes et al. ${ }^{2}$ Shape assessment was performed using 3D reconstructions of the MRA and CTA images, which were previously shown to adequately assess aneurysm shape..$^{5,19}$

\section{Statistical Analysis}

Descriptive statistics were used to describe the baseline characteristics for the group of patients with stable aneurysms and for the group with aneurysm growth or rupture. The Student t-test or Mann-Whitney U-test was used as appropriate to evaluate differences in baseline characteristics for continuous variables. Fisher's exact test was used for categorical variables. We calculated relative risks with $95 \%$ confidence intervals to identify factors associated with an increased likelihood of aneurysm growth or rupture during short-term follow-up; $\mathrm{p}<0.05$ was used as the limit for statistical significance. We used IBM SPSS (version 23.0, IBM Corp.) to perform the analyses.

\section{Results}

\section{Baseline Characteristics}

A total of 206 patients with 267 aneurysms met the inclusion criteria (Table 1). Twenty-two of the 206 patients had died by the time this study was conducted. Of these, 2 patients died of an SAH outside the short-term follow-up interval, 1 patient died of an SAH from an additional previously electively coiled aneurysm, and 1 patient died suddenly, potentially due to aneurysm rupture. The remaining 18 patients died of reasons unrelated to the intracranial aneurysm. Sixty-eight percent of patients who were still alive returned the questionnaire. The median age at initial presentation was 56 years (range 28-79 years), and the median interval between diagnosis and short-term followup was 1 year (range $0.3-2$ years). The mean aneurysm size at initial evaluation was $4.7 \mathrm{~mm}(\mathrm{SD} 2.8 \mathrm{~mm})$. 


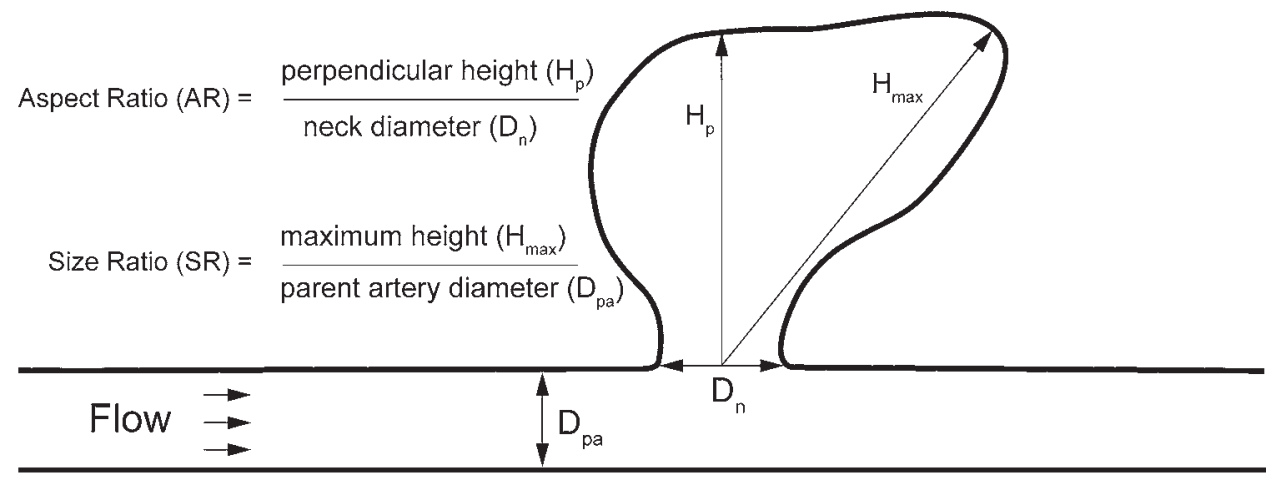

FIG. 1. Illustration of the definitions of aspect ratio and size ratio.

\section{Aneurysm Growth}

Seven of 267 aneurysms $(2.6 \%, 95 \%$ CI $1.2 \%-5.6 \%)$ enlarged during short-term follow-up (Table 2). Growth was detected after a median follow-up period of 1.1 years (range $0.8-1.6$ years). Five patients were electively treated after growth was detected. One patient declined treatment. The remaining patient was not offered treatment, since growth was not detected by the radiologist during followup. However, during this study we detected the formation of a bleb during follow-up, based on which we described the aneurysm as enlarged. Treatment-associated complications occurred in 1 patient treated with stent-assisted coiling. This patient suffered an in-stent thrombosis shortly after discharge, with persistent neurological impairment at follow-up.

\section{Aneurysm Rupture}

One of 267 aneurysms $(0.4 \%, 95 \% \mathrm{CI} 0.0 \%-2.4 \%)$ ruptured 10 months after initial discovery (case 2). It was an anterior communicating artery aneurysm in a 79-year-old man with a maximum diameter of $22 \mathrm{~mm}$ at diagnosis. The aneurysm was left untreated at initial presentation due to the patient's age in combination with extensive comorbidities and intraaneurysmal thrombosis increasing the treatment-associated risks. At the time of rupture, the maximum diameter had increased to $30 \mathrm{~mm}$. The patient did not survive the rupture.

\section{Risk Factors}

Patients with aneurysm growth or rupture were older than patients with stable aneurysms (median age 64 vs 56 years, $p=0.04$ ). The presence of ADPKD was statistically significant for a higher likelihood of growth or rupture during short-term follow-up (RR 8.3, 95\% CI 2.0-34.7), as well as an aspect ratio $>1.6$ or size ratio $>3$ (RR 10.8, $95 \%$ CI 2.2-52.2), and an initial size $\geq 7 \mathrm{~mm}$ (RR 10.7, $95 \%$ CI $2.7-42.8)$.

\section{Discussion}

In this study, we found that aneurysm growth during short-term follow-up does occur in a small proportion of aneurysms (2.6\%). The majority of aneurysm growth and the single aneurysm rupture occurred in aneurysms larger than $7 \mathrm{~mm}$. In addition to initial size, ADPKD and
TABLE 1. Patient- and aneurysm-specific baseline characteristics

\begin{tabular}{|c|c|c|}
\hline & No Growth & Growth/Rupture \\
\hline \multicolumn{3}{|l|}{ Patient characteristics } \\
\hline No. of patients & 198 & 8 \\
\hline Median age, yrs (range) & $56(28-77)$ & $64(54-79)$ \\
\hline Female sex & $147(74)$ & $6(75)$ \\
\hline Previous SAH & $81(41)$ & $1(13)$ \\
\hline Hypertension & $89(45)$ & $5(63)$ \\
\hline Current smoking & $85(43)$ & $3(38)$ \\
\hline Acetylsalicylic acid use & $47(24)$ & $4(50)$ \\
\hline Multiplicity & $111(56)$ & $2(25)$ \\
\hline Familial intracranial aneurysms & $13(7)$ & $0(0)$ \\
\hline ADPKD & $6(3)$ & $2(25)$ \\
\hline Alcohol abuse & $6(3)$ & $1(13)$ \\
\hline \multicolumn{3}{|l|}{ Aneurysm characteristics } \\
\hline No. of aneurysms & 259 & 8 \\
\hline \multicolumn{3}{|l|}{ Initial size, $\mathrm{mm}$} \\
\hline $1.0-2.9$ & $76(29)$ & $1(13)$ \\
\hline $3.0-4.9$ & $108(42)$ & $1(13)$ \\
\hline $5.0-6.9$ & $44(17)$ & $1(13)$ \\
\hline $7.0-9.9$ & $23(9)$ & $2(25)$ \\
\hline$\geq 10.0$ & $8(3)$ & $3(38)$ \\
\hline Irregular shape & $21(8)$ & $2(25)$ \\
\hline Aspect ratio $>1.6$ or size ratio $>3$ & $52(20)$ & $6(75)$ \\
\hline \multicolumn{3}{|l|}{ Location } \\
\hline Anterior cerebral arteries* & $47(18)$ & $2(25)$ \\
\hline MCA & $123(48)$ & $4(50)$ \\
\hline Posterior communicating artery & $18(7)$ & $0(0)$ \\
\hline Internal carotid artery & $50(19)$ & $0(0)$ \\
\hline Posterior circulation† & $29(11)$ & $2(25)$ \\
\hline
\end{tabular}

$\mathrm{MCA}=$ middle cerebral artery.

Data are presented as number (\%) unless indicated otherwise.

* Includes the anterior communicating artery and anterior cerebral arteries.

$\dagger$ Includes vertebrobasilar complex and its branches. 
TABLE 2. Characteristics of unstable aneurysms

\begin{tabular}{ccccccl}
\hline Case No. & $\begin{array}{c}\text { Age (yrs), } \\
\text { Sex }\end{array}$ & Aneurysm Location & $\begin{array}{c}\text { Max Diameter at } \\
\text { Detection }(\mathrm{mm})\end{array}$ & $\begin{array}{c}\text { Extent of Growth } \\
(\mathrm{mm})^{*}\end{array}$ & $\begin{array}{c}\text { FU Duration } \\
(\mathrm{mos})\end{array}$ & Outcome \\
\hline 1 & $60, \mathrm{~F}$ & Basilar & 8.1 & 1.1 & 19 & Coiling \\
\hline 2 & $79, \mathrm{M}$ & ACoA & 22.0 & 8.0 & 10 & Rupture \\
\hline 3 & $67, \mathrm{~F}$ & Pericallosal & 6.3 & 2.8 & 13 & Coiling \\
\hline 4 & $55, \mathrm{~F}$ & MCA & 4.1 & Bleb formation & 12 & Observation \\
\hline 5 & $64, \mathrm{~F}$ & Basilar & 8.2 & 1.2 & 13 & Observation \\
\hline 6 & $63, \mathrm{~F}$ & MCA & 8.8 & $0.7 \dagger$ & 12 & Clipping \\
\hline 7 & $54, \mathrm{~F}$ & MCA & 1.9 & 1.2 & 14 & Clipping \\
\hline 8 & $69, \mathrm{M}$ & MCA & 13.1 & 4.9 & 10 & Stent-assisted coiling \\
\hline
\end{tabular}

$\mathrm{ACoA}=$ anterior communicating artery; $\mathrm{FU}=$ follow-up; $\max =$ maximum.

* Representing the maximum extent of growth, measured in one of the 3 dimensions.

† Combined with a change in morphology.

an aspect ratio $>1.6$ or size ratio $>3$ were associated with an increased likelihood of aneurysm growth or rupture. Enlarged aneurysms were nearly always electively treated soon after detection. Thus, short-term follow-up facilitates the detection of around 2 to 3 high-risk aneurysms for every 100 patients with a diagnosed unruptured intracranial aneurysm.

Two other studies investigated the yield of short-term follow-up for unruptured intracranial aneurysms during a relatively short follow-up period..$^{10,25}$ Wermer et al. evaluated the yield of short-term screening in unruptured aneurysms smaller than $5 \mathrm{~mm}$ that were detected during screening after an SAH or because of the presence of familial aneurysms. ${ }^{25}$ They identified growth in 3 (2.4\%) of 125 aneurysms during a median follow-up duration of 1.3 years (range $0.7-3.8$ years). One (0.8\%) previously ruptured aneurysm reruptured during follow-up of additional unruptured aneurysms. A previous history of both SAH and the presence of familial aneurysms were found to be associated with an increased risk of growth or rupture during short-term follow-up. In contrast to our study, none of the aneurysms were treated, since growth was only very small. The authors argued that, given the low occurrence of growth, and the absence of clinical consequences in their cohort, short-term follow-up is not useful. In our study, we demonstrated that considerable aneurysm growth also occurs in relatively small aneurysms, underlining the clinical relevance of short-term follow-up, also for smaller aneurysms. Inoue et al. found a lower incidence of growth in $14(1.1 \%)$ and rupture in $4(0.3 \%)$ of 1325 aneurysms during a median follow-up duration of 1.0 year (range $0.4-1.9$ years). ${ }^{10}$ Growth or rupture regularly occurred in aneurysms smaller than $5 \mathrm{~mm}$, with even $44 \%$ of enlarging aneurysms being smaller than $3 \mathrm{~mm}$ at initial presentation. The only variable linked to an increased risk of growth was female sex. The majority of aneurysms were treated after detection of growth. The difference in frequency of aneurysm growth between both aforementioned studies may be partially attributed to the use of different measurement protocols and criteria for aneurysm growth. Wermer et al. systematically measured aneurysms in 2 directions and noted growth as an increase of at least $0.5 \mathrm{~mm}$ in at least one direction, while Inoue et al. only measured the maximum diameter, used low-resolution MRA, and consequently described aneurysms as enlarged after an increase in maximum diameter of least 1.5 times or after a change in aneurysm morphology.

\section{Study Strengths and Limitations}

Strengths of this study include the use of a strict measurement protocol to detect aneurysm growth during a predefined short-term follow-up period. Also, all patients referred to our center with an indication for follow-up imaging are routinely recommended follow-up imaging after 1 year, limiting the risk of a selection bias among conservatively treated aneurysms in our cohort. In addition, we calculated aspect and size ratios for all aneurysms; these calculations have not been described in previous studies regarding shortterm follow-up but were shown to be associated with an increased likelihood of aneurysm growth or rupture in our study. Several limitations should also be addressed. The major limitation of this study is the retrospective nature of the data collection, which can potentially lead to misclassification of patients with regard to the presence or absence of specific risk factors. For a proportion of patients, mainly those who previously experienced an SAH, change in aneurysm size was assessed using multiple imaging modalities. Recent research showed no significant inter- and intraobserver variability between CTA- and MRA-based aneurysm size measurements. ${ }^{13}$ This suggests that changes in aneurysm size can be accurately assessed between the two different imaging modalities. Also, although we described aneurysm growth in a relatively large cohort, we were unable to perform multivariate statistical analysis due to the small number of growing aneurysms and aneurysm rupture that occurred during follow-up. ${ }^{23}$ Furthermore, we included patients with and without a history of a previous SAH, although the mechanisms underlying aneurysm progression may be different for these groups. However, although around $40 \%$ of the patients in our cohort previously experienced an $\mathrm{SAH}$, this was not associated with a statistically significant higher likelihood of aneurysm growth.

\section{Conclusions}

Growth of unruptured intracranial aneurysms occurs 
during short-term follow-up. As aneurysm growth is associated with an increased risk of rupture, we believe that short-term follow-up is clinically relevant and has an important role in reducing the risk of a potential SAH.

\section{References}

1. Backes D, Rinkel GJE, Greving JP, Velthuis BK, Murayama $\mathrm{Y}$, Takao $\mathrm{H}$, et al: ELAPSS score for prediction of risk of growth of unruptured intracranial aneurysms. Neurology 88:1600-1606, 2017

2. Backes D, Vergouwen MDI, Velthuis BK, van der Schaaf IC, Bor ASE, Algra A, et al: Difference in aneurysm characteristics between ruptured and unruptured aneurysms in patients with multiple intracranial aneurysms. Stroke 45:1299-1303, 2014

3. Bos D, Poels MM, Adams HH, Akoudad S, Cremers LG, Zonneveld HI, et al: Prevalence, clinical management, and natural course of incidental findings on brain MR images: the population-based Rotterdam Scan Study. Radiology 281:507-515, 2016

4. Dhar S, Tremmel M, Mocco J, Kim M, Yamamoto J, Siddiqui AH, et al: Morphology parameters for intracranial aneurysm rupture risk assessment. Neurosurgery 63:185-197, 2008

5. Franklin B, Gasco J, Uribe T, VonRitschl RHA, Hauck E: Diagnostic accuracy and inter-rater reliability of 64-multislice 3D-CTA compared to intra-arterial DSA for intracranial aneurysms. J Clin Neurosci 17:579-583, 2010

6. Gondar R, Gautschi OP, Cuony J, Perren F, Jägersberg M, Corniola MV, et al: Unruptured intracranial aneurysm follow-up and treatment after morphological change is safe: observational study and systematic review. J Neurol Neurosurg Psychiatry 87:1277-1282, 2016

7. Greving JP, Wermer MJ, Brown RD Jr, Morita A, Juvela S, Yonekura M, et al: Development of the PHASES score for prediction of risk of rupture of intracranial aneurysms: a pooled analysis of six prospective cohort studies. Lancet Neurol 13:59-66, 2014

8. Güresir E, Vatter H, Schuss P, Platz J, Konczalla J, de Rochement Rdu M, et al: Natural history of small unruptured anterior circulation aneurysms: a prospective cohort study. Stroke 44:3027-3031, 2013

9. Harris PA, Taylor R, Thielke R, Payne J, Gonzalez N, Conde JG: Research electronic data capture (REDCap) - a metadata-driven methodology and workflow process for providing translational research informatics support. J Biomed Inform 42:377-381, 2009

10. Inoue T, Shimizu H, Fujimura M, Saito A, Tominaga T: Annual rupture risk of growing unruptured cerebral aneurysms detected by magnetic resonance angiography. J Neurosurg 117:20-25, 2012

11. Ishibashi T, Murayama Y, Urashima M, Saguchi T, Ebara M, Arakawa H, et al: Unruptured intracranial aneurysms: incidence of rupture and risk factors. Stroke 40:313-316, 2009

12. Jeon JS, Ahn JH, Huh W, Son YJ, Bang JS, Kang HS, et al: A retrospective analysis on the natural history of incidental small paraclinoid unruptured aneurysm. J Neurol Neurosurg Psychiatry 85:289-294, 2014

13. Kim HJ, Yoon DY, Kim ES, Lee HJ, Jeon HJ, Lee JY, et al: Intraobserver and interobserver variability in CT angiography and MR angiography measurements of the size of cerebral aneurysms. Neuroradiology 59:491-497, 2017

14. Matsubara S, Hadeishi H, Suzuki A, Yasui N, Nishimura $\mathrm{H}$ : Incidence and risk factors for the growth of unruptured cerebral aneurysms: observation using serial computerized tomography angiography. J Neurosurg 101:908-914, 2004
15. Matsumoto K, Oshino S, Sasaki M, Tsuruzono K, Taketsuna $\mathrm{S}$, Yoshimine T: Incidence of growth and rupture of unruptured intracranial aneurysms followed by serial MRA. Acta Neurochir (Wien) 155:211-216, 2013

16. Mehan WAJ Jr, Romero JM, Hirsch JA, Sabbag DJ, Gonzalez RG, Heit JJ, et al: Unruptured intracranial aneurysms conservatively followed with serial CT angiography: could morphology and growth predict rupture? J Neurointerv Surg 6:761-766, 2014

17. Nieuwkamp DJ, Setz LE, Algra A, Linn FH, de Rooij NK, Rinkel GJ: Changes in case fatality of aneurysmal subarachnoid haemorrhage over time, according to age, sex, and region: a meta-analysis. Lancet Neurol 8:635-642, 2009

18. Phan TG, Huston J III, Brown RD Jr, Wiebers DO, Piepgras DG: Intracranial saccular aneurysm enlargement determined using serial magnetic resonance angiography. J Neurosurg 97:1023-1028, 2002

19. Okahara M, Kiyosue H, Yamashita M, Nagatomi H, Hata H, Saginoya T, et al: Diagnostic accuracy of magnetic resonance angiography for cerebral aneurysms in correlation with 3Ddigital subtraction angiographic images: a study of 133 aneurysms. Stroke 33:1803-1808, 2002

20. Sonobe M, Yamazaki T, Yonekura M, Kikuchi H: Small unruptured intracranial aneurysm verification study: SUAVe study, Japan. Stroke 41:1969-1977, 2010

21. Ujiie H, Tachibana H, Hiramatsu O, Hazel AL, Matsumoto T, Ogasawara Y, et al: Effects of size and shape (aspect ratio) on the hemodynamics of saccular aneurysms: a possible index for surgical treatment of intracranial aneurysms. Neurosurgery 45:119-130, 1999

22. Villablanca JP, Duckwiler GR, Jahan R, Tateshima S, Martin NA, Frazee J, et al: Natural history of asymptomatic unruptured cerebral aneurysms evaluated at CT angiography: growth and rupture incidence and correlation with epidemiologic risk factors. Radiology 269:258-265, 2013

23. Vittinghoff E, McCulloch CE: Relaxing the rule of ten events per variable in logistic and Cox regression. Am J Epidemiol 165:710-718, 2007

24. Vlak MH, Algra A, Brandenburg R, Rinkel GJ: Prevalence of unruptured intracranial aneurysms, with emphasis on sex, age, comorbidity, country, and time period: a systematic review and meta-analysis. Lancet Neurol 10:626-636, 2011

25. Wermer MJH, van der Schaaf IC, Velthuis BK, Majoie CB, Albrecht KW, Rinkel GJE: Yield of short-term follow-up CT/ MR angiography for small aneurysms detected at screening. Stroke 37:414-418, 2006

\section{Disclosures}

The authors report no conflict of interest concerning the materials or methods used in this study or the findings specified in this paper.

\section{Author Contributions}

Conception and design: Molenberg, Aalbers, Uyttenboogaart, van Dijk. Acquisition of data: Molenberg, Aalbers, Uyttenboogaart. Analysis and interpretation of data: all authors. Drafting the article: Molenberg, Aalbers. Critically revising the article: all authors. Reviewed submitted version of manuscript: all authors. Approved the final version of the manuscript on behalf of all authors: Molenberg. Statistical analysis: Molenberg. Study supervision: van Dijk.

\section{Correspondence}

Rob Molenberg: University Medical Center Groningen, The Netherlands.r.molenberg@umcg.nl. 